\title{
Order and disorder in the local and long-range structure of the spin-glass pyrochlore, $\mathrm{Tb}_{2} \mathrm{Mo}_{2} \mathrm{O}_{7}$
}

\author{
Yu Jiang ${ }^{1}$, Ashfia Huq ${ }^{2}$, Corwin H. Booth ${ }^{1}$, Georg Ehlers ${ }^{2}$, John E. \\ Greedan $^{3}$, Jason S. Gardner ${ }^{4,5}$ \\ ${ }^{1}$ Chemical Sciences Division, Lawrence Berkeley National Laboratory, Berkeley, CA 94720, USA \\ ${ }^{2}$ Neutron Scattering Science Division, Oak Ridge National Laboratory, Oak Ridge, TN \\ 37831-6475, USA \\ ${ }^{3}$ Department of Chemistry and Brockhouse Institute for Materials Research, McMaster \\ University, Hamilton, Ontario, L8S 4M1, Canada \\ ${ }^{4}$ Department of Physics, Indiana University, Bloomington, IN 47408, USA \\ ${ }^{5}$ NIST Center for Neutron Research, NIST, Gaithersburg, MD 20899-6102, USA \\ E-mail: chbooth@lbl.gov
}

Date: 14 February 2011

\begin{abstract}
.
To understand the origin of the spin-glass state in molybdate pyrochlores, the structure of $\mathrm{Tb}_{2} \mathrm{Mo}_{2} \mathrm{O}_{7}$ is investigated using two techniques: the long-range lattice structure was measured using neutron powder diffraction (NPD), and local structure information was obtained from the extended x-ray absorption fine structure (EXAFS) technique. While the long-range structure appears generally well ordered, enhanced mean-squared site displacements on the $O(1)$ site and the lack of temperature dependence of the strongly anisotropic displacement parameters for both the $\mathrm{Mo}$ and $\mathrm{O}(1)$ sites indicate some disorder exists. Likewise, the local structure measurements indicate some Mo-Mo and $\mathrm{Tb}-\mathrm{O}(1)$ nearest-neighbor disorder exists, similar to that found in the related spin-glass pyrochlore, $\mathrm{Y}_{2} \mathrm{Mo}_{2} \mathrm{O}_{7}$. Although the freezing temperature in $\mathrm{Tb}_{2} \mathrm{Mo}_{2} \mathrm{O}_{7}, 25 \mathrm{~K}$, is slightly higher than in $\mathrm{Y}_{2} \mathrm{Mo}_{2} \mathrm{O}_{7}, 22 \mathrm{~K}$, the degree of local pair distance disorder is actually less in $\mathrm{Tb}_{2} \mathrm{Mo}_{2} \mathrm{O}_{7}$. This apparent contradiction is considered in light of the interactions involved in the freezing process.
\end{abstract}

\section{Introduction}

The pyrochlore family $\left(A_{2} B_{2} \mathrm{O}_{7}\right.$, space group $F d \overline{3} m$, $A$, at $16 d$, and $B$, at $16 c$ site, representing different transition or rare earth metal ions) has attracted much attention over the past few decades. 
Pyrochlores have an unusual lattice structure: $A$ and $B$ ions reside on two distinct interpenetrating lattices of corner-sharing tetrahedra, with oxygens surrounding each metal ion. If the ions on either site have a magnetic moment, and the nearest-neighbor exchange interaction is antiferromagnetic (AFM), the system is subject to geometric magnetic frustration, which may lead to the absence of long-range magnetic order at finite temperature, such as occurs in the spin-liquid and spin-ice states [1]. Together with large lattice disorder, the spin-glass (SG) state may appear [2].

Among the roughly one hundred pyrochlores synthesized so far, the $A_{2} \mathrm{Mo}_{2} \mathrm{O}_{7}$ system has received particular attention, since these materials span a wide variety of ground states due to the link between the ionic radius of the $A$-site ion and the electrical transport properties, which in turn are strongly correlated to the magnetic properties. For example, as the ionic radius of the $A$-site ion decreases from $\mathrm{Nd}(\sim 1.11 \AA)$ to $\mathrm{Lu}(\sim 0.98 \AA)$, the ground state changes from a ferromagnetic metal to a non-ferromagnetic insulator at $\mathrm{Tb}$ (ionic radius $\sim 1.04 \AA$ ) [3]. $\mathrm{Tb}_{2} \mathrm{Mo}_{2} \mathrm{O}_{7}$ and $\mathrm{Y}_{2} \mathrm{Mo}_{2} \mathrm{O}_{7}$ (Y, ionic radius $\sim 1.02 \AA$ ) are particularly important, since they are the first two spin glasses found in the pyrochlore family (SG transition temperatures, $T_{S G}, \sim 25$ and $22 \mathrm{~K}$, respectively). Although both of them are on the insulating non-ferromagnetic side of the phase diagram near the metallic/insulating boundary, their Curie-Weiss temperatures are quite different: $\theta_{C W} \sim 20 \mathrm{~K}$ for $\mathrm{Tb}_{2} \mathrm{Mo}_{2} \mathrm{O}_{7}$, and $\theta_{C W} \sim-60 \mathrm{~K}$ for $\mathrm{Y}_{2} \mathrm{Mo}_{2} \mathrm{O}_{7} .{ }^{1}$ The large difference is thought to be caused by a ferromagnetic interaction between $A$-site ions, which may play an important role in the formation of the SG state.

The strong similarities between $\mathrm{Tb}_{2} \mathrm{Mo}_{2} \mathrm{O}_{7}$ and $\mathrm{Y}_{2} \mathrm{Mo}_{2} \mathrm{O}_{7}$ warrant a close examination of the properties of the more-studied $\mathrm{Y}_{2} \mathrm{Mo}_{2} \mathrm{O}_{7}$. Mo is the only magnetic ion in $\mathrm{Y}_{2} \mathrm{Mo}_{2} \mathrm{O}_{7}$, and has an effective moment of $2.55 \mu_{B}$, close to the expected value $2.8 \mu_{B}$ for $S=1 \mathrm{Mo}^{4+}$ [4]. A strong AFM coupling between the nearest-neighbor Mo ions is deduced from magnetization measurements and confirmed in neutron diffraction measurements, where the vector sum of the moments on each tetrahedron was shown to equal zero [4]. Nuclear magnetic resonance (NMR) measurements on ${ }^{89} \mathrm{Y}$ [5] show multiple discrete values of the local susceptibility at the $\mathrm{Y}$ sites in the $\mathrm{Y}_{2} \mathrm{Mo}_{2} \mathrm{O}_{7}$ material, indicative of the local magnetic disorder expected in a SG state [6]. This result is supported by $\mu \mathrm{SR}$ measurements $[7,8]$ which suggest a broad distribution of the internal magnetic field, inconsistent with a narrow distribution of magnetic interactions.

Local probes of the crystal structure also indicate disorder; however, there is some disagreement regarding the exact nature of this disorder. An extended x-ray absorption fine structure (EXAFS) study of both Y and Mo $K$-edge absorption spectra [9] suggests that the primary disorder involves the Mo atoms displacing in a direction that is roughly parallel to the local Mo-Mo pairs and perpendicular to the Mo-Y pairs, with the magnitude of the displacement varying throughout the solid. These measurements also indicate that the $\mathrm{O}(1)$ octahedron surrounding Mo is only slightly disordered, while the $\mathrm{O}(1)$ pairs are more disordered, consistent with $\mathrm{MoO}(1)_{6}$ displacing as a unit. A recent study involving both neutron powder diffraction (NPD) and neutron pair distribution function (NPDF) experiments also found evidence for disorder [10]. Anisotropic displacement parameters (ADPs) for both the Mo and $\mathrm{O}(1)$ atoms were found to be anomalously large compared to those for isostructural $\mathrm{Y}_{2} \mathrm{Sn}_{2} \mathrm{O}_{7}$ and showed unusual temperature dependences. Simple inspection of the real space pair-distribution function, $G(r)$, focused attention on the Y$\mathrm{O}(1)$ pairs as a major source of disorder. Fits to the $G(r)$ using both a dynamic disorder model (all

1 The $\theta_{C W}$ for the $\mathrm{Y}_{2} \mathrm{Mo}_{2} \mathrm{O}_{7}$, as reported in many other papers, is obtained by fitting the magnetic susceptibility data up to $\sim 300 \mathrm{~K}$; if the data are fit to $800 \mathrm{~K}, \theta_{C W} \sim-200 \mathrm{~K}[3]$. 
atoms with ADPs) and a model involving a statically split $\mathrm{O}(1)$ site gave satisfactory agreement with the observed data. As well, from the split site model it was possible to estimate the disorderinduced distribution of nearest neighbor Mo-O-Mo superexchange interactions, $\Delta J$, which was found to satisfy the criterion of Saunders and Chalker for stabilization of the spin glass ground state in "weakly disordered" pyrochlore systems [11]. A major role for Mo-Mo disorder was not found in the NPD/NPDF studies. Nonetheless, there are areas of significant agreement with the EXAFS results. For example, in the NPDF work, the Y-O(1) pair distance is split by $\Delta R=0.132$ $\AA$. These PDF data have better resolving power for such a split than the EXAFS data; however, an unresolved split pair distance would manifest in the latter as a so-called "static" contribution to the mean-squared pair distance displacement parameter, $\sigma_{\text {stat }}^{2}=(\Delta R / 2)^{2}=0.0042 \AA^{2}$. The temperature dependence of the EXAFS data were, in fact, used to obtain $\sigma_{\text {stat }}^{2}=0.0046 \AA^{2}$, and so the PDF and EXAFS results are in good quantitative agreement. Likewise, the PDF measurement measured a split in the Mo-O $(1)$ pair of $\Delta R=0.080 \AA$, which would correspond to a $\sigma_{\text {stat }}^{2}=0.0016$ $\AA^{2}$, again consistent with the EXAFS result of $\sigma_{\text {stat }}^{2}=0.0024 \AA^{2}$ for this pair. The PDF and EXAFS results are therefore in excellent agreement regarding the $\mathrm{Y}-\mathrm{O}(1)$ and $\mathrm{Mo}-\mathrm{O}(1)$ disorder. However, the PDF fit model doesn't support large Mo-Mo pair-distance disorder. This aspect of these measurements remains unreconciled and more studies are warranted.

The magnetic interactions are more complicated in $\mathrm{Tb}_{2} \mathrm{Mo}_{2} \mathrm{O}_{7}$, because there is another magnetic ion, Tb, at the $A$ site. To understand the origin of the $\mathrm{SG}$ behavior of $\mathrm{Tb}_{2} \mathrm{Mo}_{2} \mathrm{O}_{7}$, a number of neutron scattering measurements have been conducted $[3,12,13]$. Taking account of the same effective moment of $\mathrm{Mo}^{4+}$ measured in $\mathrm{Y}_{2} \mathrm{Mo}_{2} \mathrm{O}_{7}$, these measurements indicate that the $\mathrm{Tb}$ ion has an effective moment of $\mu_{e f f} \approx 8.83 \mu_{B}$ (close to $\mathrm{Tb}^{3+}, L=3, J=6$ ), which is three times larger than that of $\mathrm{Mo}^{4+}$. In addition, the magnetic diffraction experiments suggest a FM coupling of $\mathrm{Tb}-\mathrm{Tb}$ up to the fourth metal-metal shell, and a strong AFM coupling for Tb-Mo in the second metal-metal shell. Due to the relatively smaller Mo moment, the magnetic correlation of Mo-Mo is not resolved by these measurements, but it is believed to be AFM as found in $\mathrm{Y}_{2} \mathrm{Mo}_{2} \mathrm{O}_{7}$ given the relatively low measured value of $\theta_{C W}$. Short range correlations were also observed in a single crystal experiment [14]. The authors suggested the Tb spin structure resembles the local spin ice structure, but the moments are tilted slightly off the local $<111>$ direction and the static moment is only $4 \mu_{B}$ at $1.6 \mathrm{~K}$, significantly less than expected for a $\mathrm{Tb}^{3+}$ ionic moment.

In addition to more complicated magnetic interactions in $\mathrm{Tb}_{2} \mathrm{Mo}_{2} \mathrm{O}_{7}$, Tb has a slightly larger ionic radius than $\mathrm{Y}$. This larger radius may alter the local distortions and the magnetic interactions between Mo ions. In a previous study, Sato et al. [15] investigated the magnetic correlation of Mo-Mo with the $A$-site ionic radius in $\mathrm{Y}_{2} \mathrm{Mo}_{2} \mathrm{O}_{7}$ by substituting $\mathrm{Y}$ with another non-magnetic ion, La. It was found that the $\theta_{C W}$ changes from $-60 \mathrm{~K}$ to $+40 \mathrm{~K}$ as the fraction of La reaches the solubility limit of $50 \%$. However, the SG phase exists through all concentrations, with only a $\pm 2 \mathrm{~K}$ variation of $T_{S G}$ across the series, even when the average $A$-site ionic radius reaches the value of $\mathrm{Sm}$ or Nd on the metallic FM side of the phase diagram. This suggests that a FM/AFM competition exists among Mo ions, and can be tuned by manipulating the average $A$-site ion radius, although the lack of long-range magnetic order and the distorted local environment in the parent compound, $\mathrm{Y}_{2} \mathrm{Mo}_{2} \mathrm{O}_{7}$, may prevent a phase transition to a long-range ordered FM state.

There are other indications of the sensitivity of the magnetic interactions to local structure differences in the pyrochlore molybdates. For instance, several research groups have recently reinvestigated the $A$-site ionic radius problem. Although a similar trend in $\theta_{C W}$ with the change 
of the average $A$-site ionic radius is found [13, 16, 17], the development of the FM phase with increasing average $A$-site ionic radius is shown to be more complicated. For example, the SG phase in $\left(\mathrm{Eu}_{1-x} \mathrm{La}_{x}\right)_{2} \mathrm{Mo}_{2} \mathrm{O}_{7}$, which has a long-range ordered FM phase $\left(T_{C} \approx 45 \mathrm{~K}\right)$, still emerges with $T_{S G} \approx 25 \mathrm{~K}$ [16], while for $\left(\mathrm{Tb}_{1-x} \mathrm{La}_{x}\right)_{2} \mathrm{Mo}_{2} \mathrm{O}_{7}$, a FM phase occurs for $x>0.05$ with no reentrant $\mathrm{SG}$ phase [13]. In addition, a ferromagnetic phase in $\mathrm{Gd}_{2} \mathrm{Mo}_{2} \mathrm{O}_{7}$ can be driven toward a SG phase with applied pressure [17].

Despite all the evidence of the importance of local lattice disorder in the molybdate pyrochlores and especially in $\mathrm{Tb}_{2} \mathrm{Mo}_{2} \mathrm{O}_{7}$, very little is known about the local structure of $\mathrm{Tb}_{2} \mathrm{Mo}_{2} \mathrm{O}_{7}$, and therefore about the relationship between lattice disorder and magnetism in that system. Here, we present temperature-dependent NPD and EXAFS data on $\mathrm{Tb}_{2} \mathrm{Mo}_{2} \mathrm{O}_{7}$, and directly compare the experimental results with those from $\mathrm{Y}_{2} \mathrm{Mo}_{2} \mathrm{O}_{7}$ to better understand the difference in their local structures and how lattice disorder affects magnetic interaction in both these SG pyrochlores.

\section{Experimental Details}

Polycrystalline $\mathrm{Tb}_{2} \mathrm{Mo}_{2} \mathrm{O}_{7}$ samples were prepared using previously reported methods [3]. The magnetic-susceptibility measurements were made with a commercially available DC superconducting interference device (SQUID) magnetometer in a small applied field, no more than $2 \mathrm{mT}$. The samples were cooled from room temperature in this field and measurements were taken whilst warming from a base temperature of $2 \mathrm{~K}$.

NPD measurements were performed at both $12 \mathrm{~K}$ and $295 \mathrm{~K}$ on a 3.5 g sample of $\mathrm{Tb}_{2} \mathrm{Mo}_{2} \mathrm{O}_{7}$ using the new POWGEN diffractometer at the Spallation Neutron Source (SNS), Oak Ridge National Laboratory. At the time, the instrument was still in its commissioning phase, and three detector banks were available (at $2 \theta=45^{\circ}, 85^{\circ}, 115^{\circ}$ ). The $d$-spacings covered by the setup range from

$d \sim 4 \AA$ to $\sim 0.3 \AA$, so the first Bragg peak in range was the (220). The raw detector counts were corrected for efficiency using data taken on a vanadium standard.

For the EXAFS measurements, two $\mathrm{Tb}_{2} \mathrm{Mo}_{2} \mathrm{O}_{7}$ samples were used. By comparing the magnetic susceptibilities of the samples, we conclude that they have the same properties within error (figure 1 ), a point further amplified below with the Mo $K$-edge EXAFS data. The $\mathrm{Tb} L_{\mathrm{III}}$ and Mo $K$-edge x-ray absorption data on the older sample (sample 1) were collected at the Stanford Synchrotron Radiation Lightsource (SSRL) SPEAR2 beamline 4-3 in June and December 2000. A half-tuned double crystal $\mathrm{Si}(220)$ monochromator with a slit height of $0.7 \mathrm{~mm}$ was used, and 2 scans each were collected for $\mathrm{Tb} L_{\mathrm{III}}$ edge at $T=20,100$ and $300 \mathrm{~K}$, and for the Mo $K$-edge only at $30 \mathrm{~K}$. The Mo $K$-edge x-ray absorption data were collected on the newer sample (sample 2, the same sample as characterized above by NPD) at the SSRL SPEAR3 beamline 4-1 in June 2010, using the same experimental setup, except with a narrower slit height of $0.5 \mathrm{~mm}$. 4 scans were collected at each temperature $T=50,100,200$, and $300 \mathrm{~K}$. The x-ray absorption data were then reduced using standard procedures $[18,19,20]$.

\section{Data analysis and results}

Rietveld refinements were carried out for all three histograms simultaneously using the FULLPROF package [21]. The total number of refined reflections was 4033. From this refinement (figure 2), we 


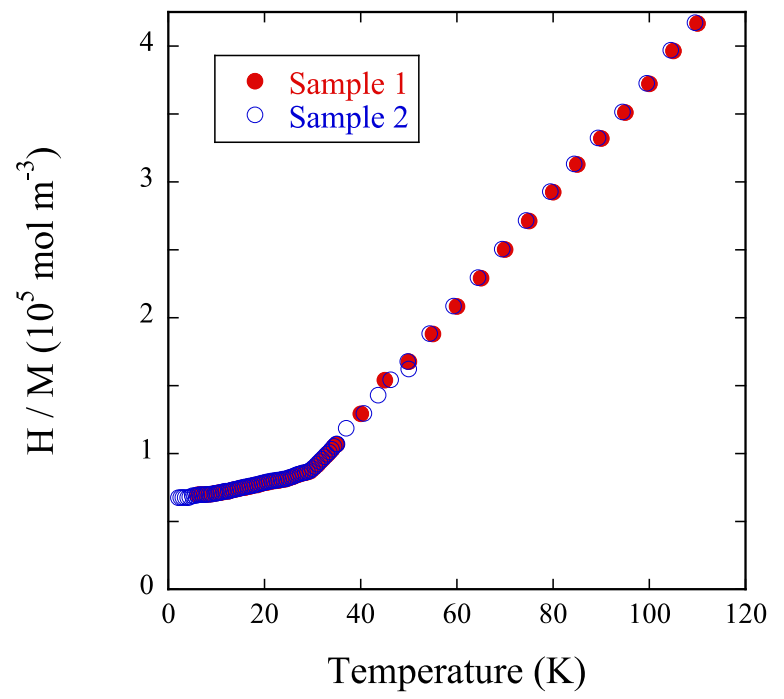

Figure 1. Comparison between the inverse magnetic susceptibilities versus temperature of the two $\mathrm{Tb}_{2} \mathrm{Mo}_{2} \mathrm{O}_{7}$ samples used in this study.

obtain the lattice parameter and $48 f x$-position (see table 1 for site definitions), occupation number, and the mean-squared displacement parameters for each site. The final results of the Rietveld refinement, including ADPs, are listed in table 2 and are compared with those for $\mathrm{Y}_{2} \mathrm{Mo}_{2} \mathrm{O}_{7}$ from previous work [10]. Note that the Mo ADP values for the Tb material are significantly smaller than those for the $\mathrm{Y}$ pyrochlore while the corresponding values for $\mathrm{O}(1)$ are more comparable at all temperatures. It is also interesting to compare the ADP for $\mathrm{Tb}_{2} \mathrm{Mo}_{2} \mathrm{O}_{7}$ with those for isostructural $\mathrm{Tb}_{2} \mathrm{Sn}_{2} \mathrm{O}_{7}$ [22] for which data are available only at $300 \mathrm{~K}$. In fact they are nearly identical, especially for the $\mathrm{Tb}$ and Mo sites, within error. There is little information regarding disorder in the stannate pyrochlore, but it is not a spin glass, showing instead a complex spin-ice behavior [23, 24]. Figure 3 shows the anisotropic displacement ellipsoids for the Mo and Tb sites for the $12 \mathrm{~K}$ data, indicating the Mo displacements are primarily into and out of the Mo tetrahedrons, mainly perpendicular to the Tb-Mo near-neighbor pairs. The total oxygen occupancy fraction is very close to 1.0, the appropriate value for the stoichiometric pyrochlore lattice. The cubic lattice constant, $a$, is close to $10.309 \AA$, the expected value for a stoichiometric pyrochlore with all the Mo in the $4+$ oxidation state [3]. These results suggest that the sample has very good crystallinity, and any foreign phase is below the detection limit.

The Tb $L_{\mathrm{III}^{-}}$and Mo $K$-edge EXAFS data were analyzed using the RSXAP package [20]. A preedge background was removed from the data to account for the background absorption from other atoms. The embedded-atom background absorption above the main absorption edge, $\mu_{0}(E)$, was estimated by fitting a 5-knot cubic spline through the data. The EXAFS oscillations were then defined as $\chi(E)=\mu(E) / \mu_{0}(E)-1$. The photoelectron wave vector was defined as $k=\sqrt{\frac{2 m\left(E-E_{0}\right)}{\hbar^{2}}}$, where $m$ is the rest mass of the photoelectron, $E$ is the incident photon energy, and $E_{0}$ is the photoelectron threshold energy, arbitrarily defined as the energy of the half-height point at the main absorption edge. The data were then fit in $r$-space to the Fourier Transform (FT) of $k^{3} \chi(k)$. 

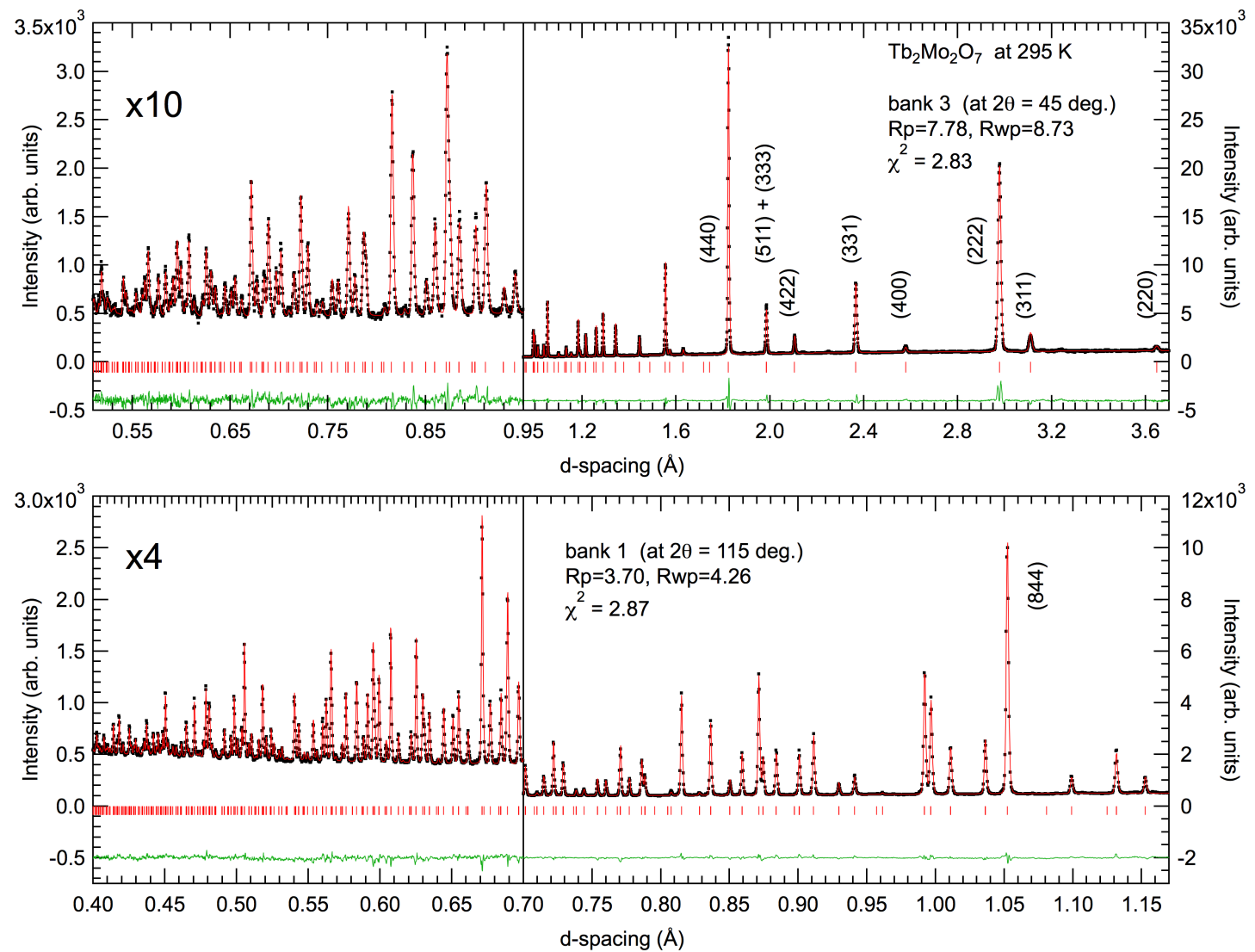

Figure 2. Room temperature $\left(T=295 \mathrm{~K}\right.$ ) neutron diffraction patterns for $\mathrm{Tb}_{2} \mathrm{Mo}_{2} \mathrm{O}_{7}$ (sample 2) from 2 of the 3 detector banks. The $\chi^{2}$ value for each bank is shown along with tic marks indicating all possible Bragg positions, the residuals, and some of the Bragg reflections are labeled. Estimated errors used in these weighted fits are based on counting statistics.

Table 1. Site definitions for the pyrochlore structure in the $F d \overline{3} m$ space group.

\begin{tabular}{lll}
\hline position & atom & $x, y, z$ \\
\hline $16 d$ & $\mathrm{~Tb} / \mathrm{Y}$ & $1 / 2,1 / 2,1 / 2$ \\
$16 c$ & $\mathrm{Mo}$ & $0,0,0$ \\
$48 f$ & $\mathrm{O}(1)$ & $x, 1 / 8,1 / 8$ \\
$8 b$ & $\mathrm{O}(2)$ & $3 / 8,3 / 8,3 / 8$ \\
\hline
\end{tabular}

Examples of the Mo $K$-edge EXAFS data are shown for the two samples in figure 4 , and of the $\mathrm{Tb}$ $L_{\mathrm{III}}{ }^{-}$and Mo $K$-edge EXAFS data in $r$-space in figure 5 . 
Table 2. Rietveld refinement results obtained by using anisotropic displacement parameters (ADPs) for NPD data of $\mathrm{Tb}_{2} \mathrm{Mo}_{2} \mathrm{O}_{7}$ (sample 2) measured at $T=12$ and $295 \mathrm{~K}$. In the refinement, the occupation numbers of metal sites are fixed at 1.0, while those of oxygen sites are allowed to vary. Values for the $U_{i j}$ ADPs in square brackets in the bottom part of the table are those for $\mathrm{Y}_{2} \mathrm{Mo}_{2} \mathrm{O}_{7}$ at comparable temperatures of $15 \mathrm{~K}$ and $300 \mathrm{~K}$ from reference [10]. Estimated parameter errors represent \pm 1 standard deviation.

\begin{tabular}{lll}
\hline parameter(site) & $12 \mathrm{~K}[15 \mathrm{~K}]$ & $295 \mathrm{~K}[300 \mathrm{~K}]$ \\
\hline lattice $a$ & $10.28481(3)$ & $10.31246(3)$ \\
$x$ & $0.3359(1)$ & $0.3356(1)$ \\
occupation $\mathrm{O}(1)$ & $0.984(4)$ & $0.992(4)$ \\
occupation $\mathrm{O}(2)$ & $1.00(1)$ & $1.00(1)$ \\
reduced $\chi^{2}$ & 1.5 & 2.4 \\
\hline$U_{11}=U_{22}=U_{33}(\mathrm{~Tb},[\mathrm{Y}])$ & $0.0020(1)[0.0044(1)]$ & $0.0062(1)[0.00086(1)]$ \\
$U_{12}=U_{13}=U_{23}(\mathrm{~Tb},[\mathrm{Y}])$ & $-0.0004(1)[-0.0014(1)]$ & $-0.0017(1)[-0.0022(1)]$ \\
$U_{11}=U_{22}=U_{33}(\mathrm{Mo},[\mathrm{Mo}])$ & $0.0061(1)[0.0109(2)]$ & $0.0054(1)[0.0114(1)]$ \\
$U_{12}=U_{13}=U_{23}(\mathrm{Mo},[\mathrm{Mo}])$ & $0.0044(2)[0.0081(2)]$ & $0.0016(2)[0.0061(1)]$ \\
$U_{11}(\mathrm{O}(1),[\mathrm{O}(1)])$ & $0.0119(2)[0.0140(2)]$ & $0.0147(2)[0.0185(2)]$ \\
$U_{22}=U_{33}(\mathrm{O}(1),[\mathrm{O}(1)])$ & $0.0044(2)[0.0065(1)]$ & $0.0069(2)[0.0096(1)]$ \\
$U_{23}(\mathrm{O}(1),[\mathrm{O}(1)])$ & $0.0005(2)[0.0016(13)]$ & $0.0026(2)[0.0016(1)]$ \\
$U_{11}=U_{22}=U_{33}(\mathrm{O}(2),[\mathrm{O}(2)])$ & $0.0031(2)[0.0030(2)]$ & $0.0050(3)[0.0056(2)]$ \\
\hline
\end{tabular}

EXAFS data such as shown in figure 5 correspond very closely to the actual radial-distribution function around the absorbing atomic species, except that the lineshape and peak position for each scattering pair are affected by the amplitude and phase of the electron scattering functions. For instance, the largest peak in figure $5 \mathrm{~b}$ at $r \approx 1.6 \AA$ corresponds to the six Mo-O(1) pairs at a bond length of $R=2.3 \AA$. In the previous EXAFS investigation on $\mathrm{Y}_{2} \mathrm{Mo}_{2} \mathrm{O}_{7}$, the FT data from both metal edges could be compared directly with little necessary recourse to detailed fits because the absorption and backscattering functions of $\mathrm{Y}$ and Mo are very similar. Unfortunately, these functions are very different for $\mathrm{Tb}$ and $\mathrm{Mo}$, and detailed fits must be performed to extract meaningful results for the Tb-Tb/Mo and Mo-Tb/Mo near-neighbor pairs.

The $r$-space EXAFS data are fit with the backscattering and phase functions calculated by FEFF 8 [26] based on the lattice structure obtained from the above NPD measurement, using a similar set of constraints as used for the EXAFS fit of $\mathrm{Y}_{2} \mathrm{Mo}_{2} \mathrm{O}_{7}$ [9]. To summarize, all single scattering pairs are included up to about $4.6 \AA$. The number of neighbors for each scattering pair was fixed to the nominal value, and a single overall scale factor, $S_{0}^{2}$, was used for each absorption edge. Multiple scattering was also included based on the structure obtained from NPD. All pair distances, $R$, and mean-squared pair distance displacements, $\sigma^{2}(T)$, are allowed to vary, except as follows. The Tb-O and Mo-O pair distances beyond $3.9 \AA$ were constrained to the change from the nominal distance of the nearest-neighbor oxygen peak. Also for the further Tb-O pairs, the $\sigma^{2}$ values were fixed to be the same, while for Mo-O pairs, the $\sigma^{2}$ values were held fixed to $0.0144 \AA^{2}$. In the Mo $K$-edge fit, we find that for $T \leq 100 \mathrm{~K}$, the Mo- $\mathrm{Tb} \sigma^{2}$ is the same as that for the Tb-Mo pair obtained from the $\mathrm{Tb} L_{\mathrm{III}}$-edge fit, as expected; above $T=100 \mathrm{~K}$, the Mo-Tb backscattering function strongly 


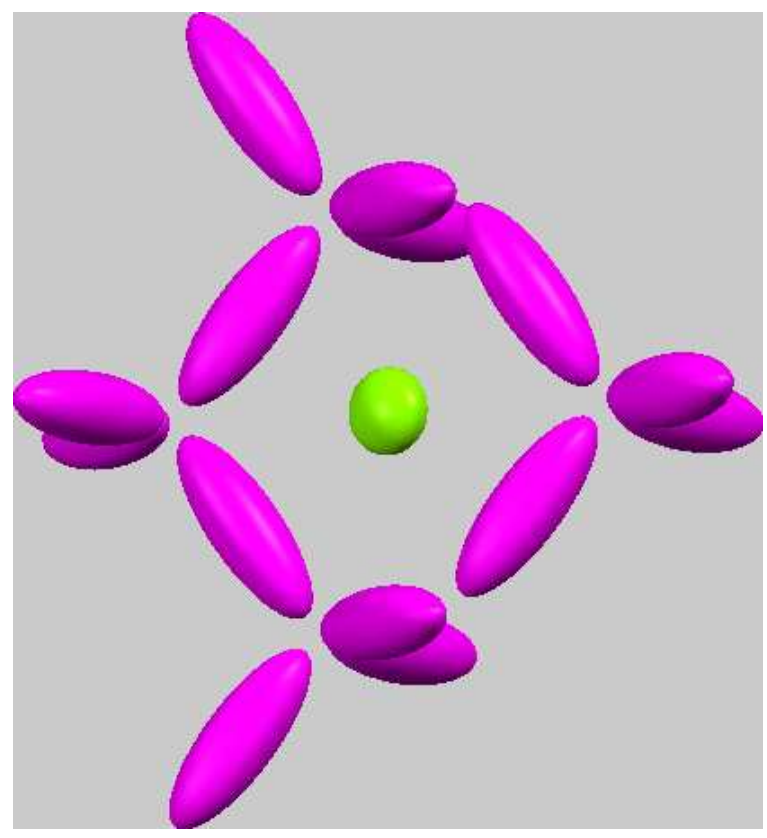

Figure 3. The $50 \%$ mean-squared displacement ellipsoids $(100 \times)$ for $\mathrm{Tb}$ (green) and Mo (magenta) from the $12 \mathrm{~K}$ data.

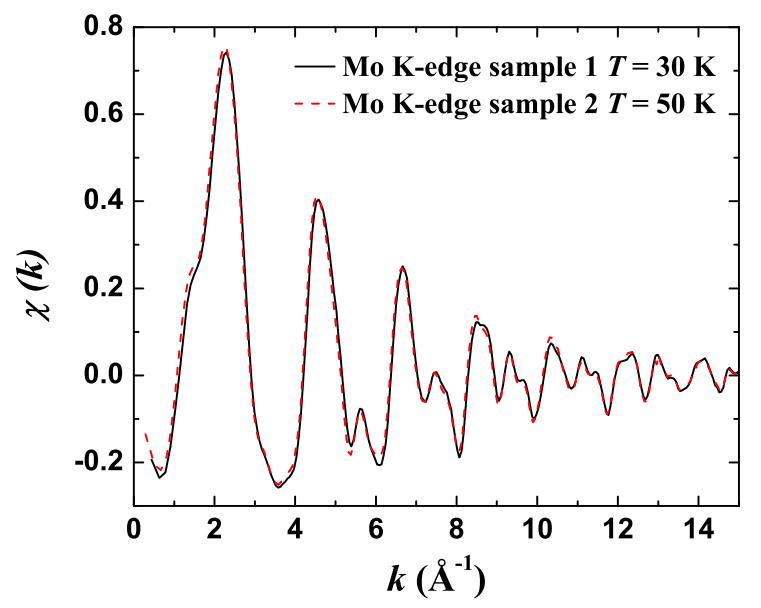

Figure 4. Mo $K$-edge data from both samples 1 and 2 as an example of data quality and to further demonstrate that the samples are identical within error.

interferes with that of Mo-Mo. Therefore, we constrain the $\sigma^{2} \mathrm{~s}$ for Mo-Tb and Tb-Mo to be the same at each temperature.

The $\sigma^{2}(T)$ results were then fit with a correlated-Debye model [27] that describes the temperature 

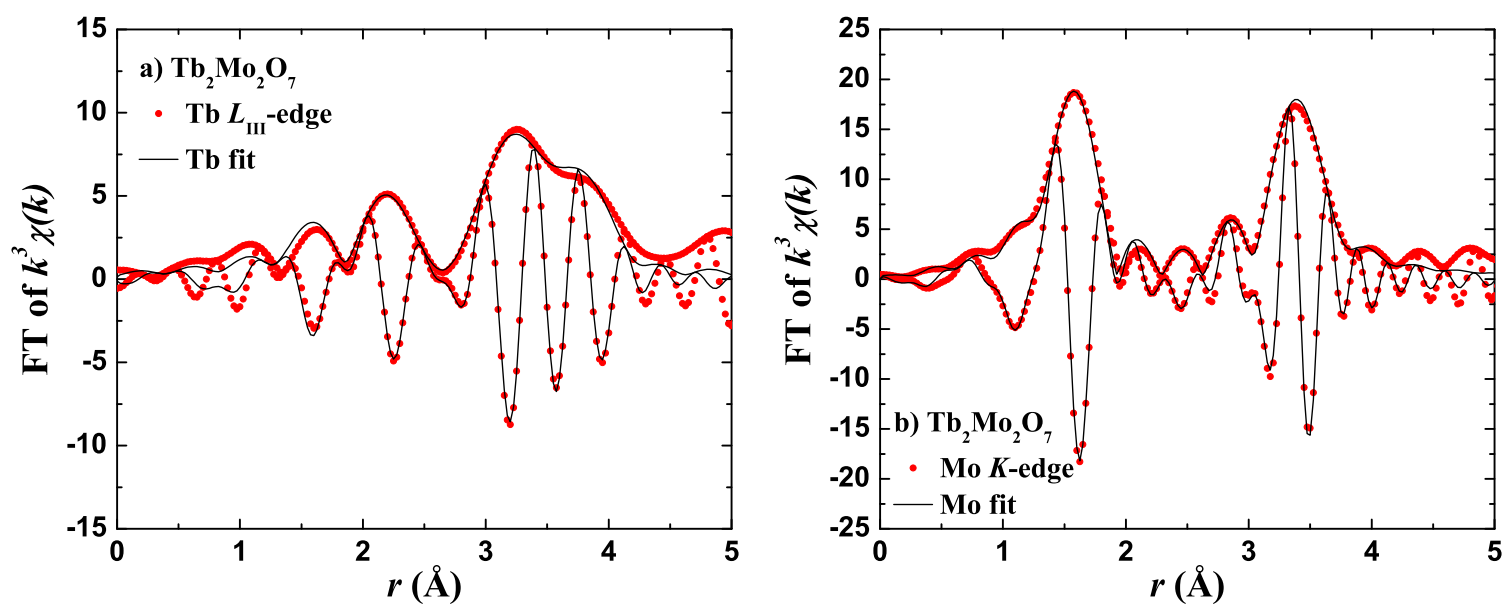

Figure 5. Fourier transform (FT) of a) Tb $L_{\mathrm{III}}$ and b) Mo $K$-edge $k^{3} \chi(k)$ data (red symbols) and fits (solid lines) for $\mathrm{Tb}_{2} \mathrm{Mo}_{2} \mathrm{O}_{7}$ (sample 1 ) at $T=20$ and $30 \mathrm{~K}$, respectively. For the $\mathrm{Tb} L_{\mathrm{III}}$-edge, the FT $k$-range is $3.0-11.0 \AA^{-1}$ with a $0.3 \AA^{-1}$ wide Gaussian window. For the Mo $K$-edge, the $k$-range is $3.0-13.8 \AA^{-1}$ with a $0.3 \AA^{-1}$ wide Gaussian window. The fit range is 1.4 to $4.2 \AA$ in both cases. The modulating line represents the real part and the outer envelope represents the amplitude $\left(\sqrt{R e^{2}+I m^{2}}\right)$ of the complex transform.
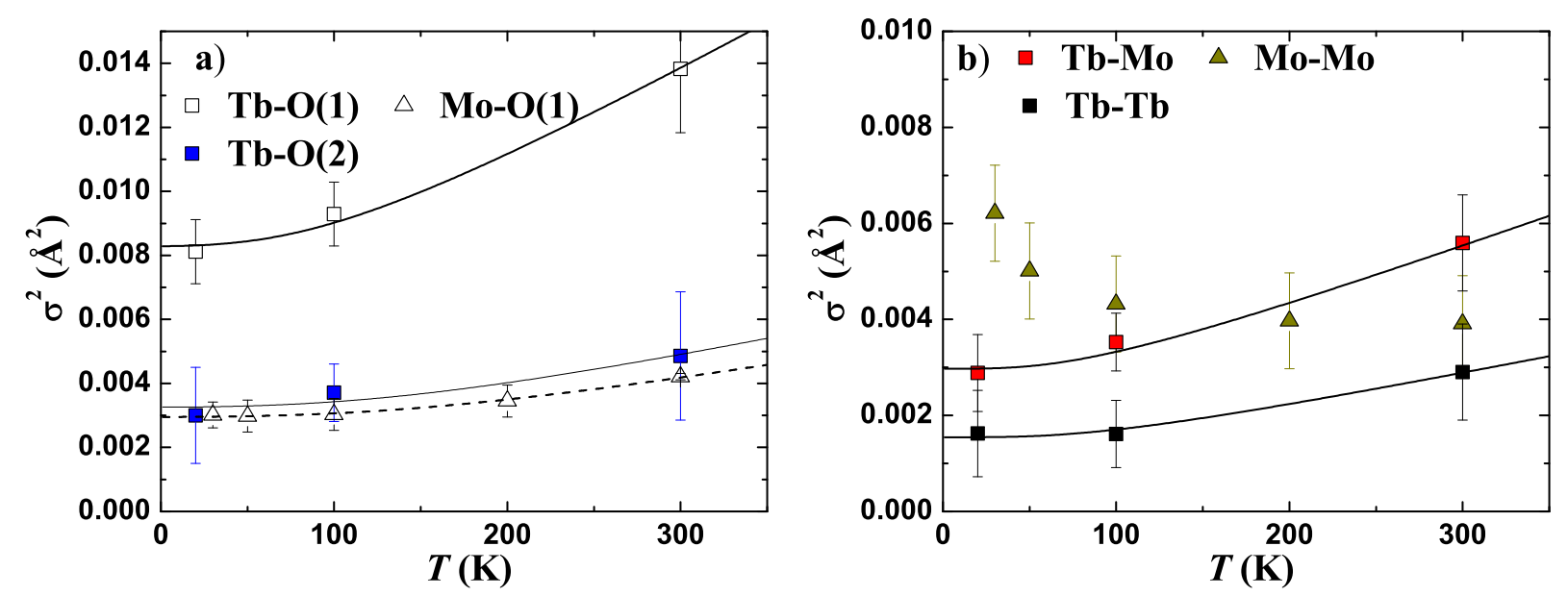

Figure 6. $\sigma^{2}(T)$ for a) metal-oxygen pairs and b) metal-metal pairs (squares for $\mathrm{Tb} L_{\mathrm{III}}$-edge data and triangles for Mo $K$-edge data) from both samples 1 and 2 (see section 2 ). Their correlatedDebye fits are also shown in each plot (solid lines for $\mathrm{Tb} L_{\mathrm{III}}$-edge data and dashed lines for Mo $K$-edge data). Note that to obtain a lower-bound estimate of the $\sigma_{\text {stat }}^{2}$, the Mo-Mo fit were held at $\Theta_{c D}=600 \mathrm{~K}$ (see section 4 for details).

dependence and the zero-point motion in terms of the correlated-Debye temperature, $\Theta_{c D}$. Any necessary offset in this model corresponds to the width of static (non-thermal) part of the pair 
Table 3. Some of the EXAFS fit results for Tb $L_{\mathrm{III}}$-edge and Mo $K$-edge data on $\mathrm{Tb}_{2} \mathrm{Mo}_{2} \mathrm{O}_{7}$. The amplitude reduction factors are $S_{0}^{2}=1.0$ for the $\mathrm{Tb} L_{\mathrm{III}}$-edge fit and 0.87 for the Mo $K$-edge fit. Results for $\sigma^{2}$ and $R$ are at $T=20 \mathrm{~K}$ for $\mathrm{Tb} L_{\mathrm{III}}$-edge and $30 \mathrm{~K}$ for Mo $K$-edge (sample 1 ), and correlated-Debye fit results are to the combined data from samples 1 and 2. EXAFS parameter error estimates are obtained using a Monte Carlo method [25]. Error estimates for $\sigma_{\text {stat }}^{2}$ and $\Theta_{c D}$ are based on the covariance matrix from the least-squares fit to $\sigma^{2}(T)$. EXAFS results reported without errors are constrained as described in the text. To obtain a lower-bound estimate of $\sigma_{\text {stat }}^{2}$ for Mo-Mo pair, $\Theta_{c D}$ is fixed at $600 \mathrm{~K}$. See text for further details.

\begin{tabular}{lcccccc}
\hline Atom pair & $N_{0}$ & $R_{\text {NPD }}(\AA)$ & $R(\AA)$ & $\sigma^{2}\left(\AA^{2}\right)$ & $\Theta_{c D}(\mathrm{~K})$ & $\sigma_{\text {stat }}^{2}\left(\AA^{2}\right)$ \\
\hline Tb-O $(2)$ & 2 & $2.23261(1)$ & $2.216(7)$ & $0.003(4)$ & $790(60)$ & $0.0004(4)$ \\
Tb-O $(1)$ & 6 & $2.4859(2)$ & $2.471(7)$ & $0.008(1)$ & $510(10)$ & $0.0037(3)$ \\
Tb-Mo & 6 & $3.64584(3)$ & $3.654(5)$ & $0.0029(6)$ & $380(20)$ & $0.0014(2)$ \\
Tb-Tb & 6 & $3.64584(3)$ & $3.664(7)$ & $0.0016(7)$ & $440(20)$ & $0.0005(1)$ \\
Tb-O(1) & 12 & $4.1718(1)$ & 4.140 & $0.010(3)$ & - & - \\
Tb-O $(1)$ & 6 & $4.6411(2)$ & 4.605 & 0.010 & - & - \\
\hline Mo-O $(1)$ & 6 & $2.0276(1)$ & $2.022(4)$ & $0.0032(4)$ & $840(20)$ & $0.0004(1)$ \\
Mo-Mo & 6 & $3.64584(3)$ & $3.68(1)$ & $0.006(1)$ & $>600$ & $\geq 0.003(1)$ \\
Mo-Tb & 6 & $3.64584(3)$ & $3.659(7)$ & 0.0029 & 380 & 0.0014 \\
Mo-O $(1)$ & 6 & $3.9160(1)$ & 3.906 & 0.0144 & - & - \\
Mo-O $(2)$ & 6 & $4.27513(3)$ & 4.264 & 0.0144 & - & - \\
Mo-O $(1)$ & 12 & $4.4127(1)$ & 4.401 & 0.0144 & - & - \\
\hline
\end{tabular}

distance distribution, $\sigma_{\text {stat }}^{2}$, and is used to quantify any potential lattice disorder.

Examples of the fits are shown in figure 5 and the results are summarized in table 3 and figure 6 .

\section{Discussion}

In principle, it should be possible to determine the degree of lattice disorder at each site by using the fitted mean-squared site displacement parameters. Making comparisons of $U$ parameters between different materials can be problematic without considering their temperature dependence. In fact, the temperature-dependent results are very illuminating. In table 2 , all of the $U$ parameters increase with temperature, as expected for thermal motion, except the Mo parameters. This same situation occurs for $\mathrm{Y}_{2} \mathrm{Mo}_{2} \mathrm{O}_{7}$ [10]. Therefore, the enhanced $U$ parameters are not due to a soft phonon mode (i.e. low Debye temperature), and can be interpreted as due to static disorder on the Mo site.

The EXAFS results are in strong agreement with these conclusions based on the NPD results. In particular, the fits show no abnormal atom-pair distances compared to the diffraction results within the estimated error. There is, however, evidence of local disorder. The Mo $K$-edge fit indicates a large $\sigma^{2}=0.006 \AA^{2}$ for Mo-Mo at $T=30 \mathrm{~K}$, that remains large at all temperatures, consistent with the NPD results for the Mo site. To obtain a lower-bound estimate of the static contribution 
to $\sigma^{2}$ of Mo-Mo, we fit to the Mo-Mo $\sigma^{2}(T)$ data with a correlated-Debye model by fixing $\Theta_{c D}=$ $600 \mathrm{~K}$, and obtain $\sigma_{\text {stat }}^{2} \geq 0.003 \AA^{2}$ (shown in table 3 ). Such a large $\sigma_{\text {stat }}^{2}$ is indicative of a large amount of pair distance disorder in this material.

Interestingly, the Mo- $\mathrm{Tb}$ (and Tb-Mo) pairs at $\sim 3.66 \AA$ only show a moderate $\sigma_{\text {stat }}^{2}=0.0014 \AA^{2}$, and the $\sigma_{\text {stat }}^{2}$ for $\mathrm{Tb}-\mathrm{Tb}$ is even smaller, indicative of a well ordered $\mathrm{Tb}_{4}$ tetrahedron. Hence, we propose that the primary direction of the disorder in the Mo-Mo pairs is roughly perpendicular to the Tb-Mo pairs and parallel to the Mo-Mo pairs, which could be caused by a varying and alternating contraction or expansion of the $\mathrm{Mo}_{4}$ tetrahedron throughout the solid, in good agreement with the Mo ADPs (figure 3). Under this assumption, and assuming the neighboring $\mathrm{Mo}_{4}$ tetrahedra contract and expand alternatively by the same amount, $\Delta R$, the six Mo-Mo pairs would split into three shorter $(R-\Delta R)$ and three longer $(R+\Delta R)$ pairs, producing a pair distance difference of $2 \Delta R \sim 0.11 \AA$ in accordance with the estimated $\sigma_{\text {stat }}^{2}$ for Mo-Mo. Attempts to fit the data to such a model are not satisfactory, leading us to the conclusion that the magnitude of $\Delta R$ may vary randomly throughout the solid.

Table 3 and figure 6 also show that the Mo-O(1) octahedron remains a tightly bound unit, with a high $\Theta_{c D}$ and little static disorder. Since the Tb-O(1) pair is significantly disordered, while the $\mathrm{Tb}_{4}$ tetrahedron is not, we conclude that the Mo disorder carries the $\mathrm{O}(1)$ octahedron with it. Similarly, the small $\sigma_{\text {stat }}^{2}$ for $\mathrm{Tb}-\mathrm{O}(2)$ suggests the $\mathrm{O}(2)$ atoms are associated with $\mathrm{Tb}$ ions. This strong affiliation of oxygen ions to their nearest metal ions, also observed in $\mathrm{Y}_{2} \mathrm{Mo}_{2} \mathrm{O}_{7}$, is probably due to strong bonding between the metal ions and their closest oxygens, which is confirmed by their high $\Theta_{c D}$.

This disordered model is similar to the one proposed based on the previous EXAFS results for the spin-glass pyrochlore $\mathrm{Y}_{2} \mathrm{Mo}_{2} \mathrm{O}_{7}$, in which even larger disorder is measured in the Mo-Mo pair $\left(\sigma_{\text {stat }}^{2} \sim 0.026 \AA^{2}\right)$ and $\mathrm{Y}-\mathrm{O}(1)$ pair [9]. In contrast, the SG transition temperatures are comparable for both the $\mathrm{Tb}_{2} \mathrm{Mo}_{2} \mathrm{O}_{7}(25 \mathrm{~K})$ and $\mathrm{Y}_{2} \mathrm{Mo}_{2} \mathrm{O}_{7}(22 \mathrm{~K})$ systems, with $T_{S G}$ slightly larger in $\mathrm{Tb}_{2} \mathrm{Mo}_{2} \mathrm{O}_{7}$. The central question therefore becomes one of identifying how such an apparent contradiction can arise. At the present time, there are two leading candidates, the first focusing on the local magnetic interactions and the second on the exact nature of the spin-glass/disorder relationship in the pyrochlores. For the first, it is important to recall that $\mathrm{Tb}_{2} \mathrm{Mo}_{2} \mathrm{O}_{7}$ and $\mathrm{Y}_{2} \mathrm{Mo}_{2} \mathrm{O}_{7}$ have different magnetic environments due to the magnetic $\mathrm{Tb}$ ion, which may indicate a different formation mechanism of the SG state in $\mathrm{Tb}_{2} \mathrm{Mo}_{2} \mathrm{O}_{7}$. Neutron scattering experiments [3] show that competition between near-neighbor AFM and FM correlations exists, consistent with the small $\theta_{C W}$. Although the Mo-Mo correlations cannot be easily obtained due to the small $\mathrm{Mo}^{4+}$ magnetic moment, they are likely AFM, but weaker compared with the Mo-Mo interaction in $\mathrm{Y}_{2} \mathrm{Mo}_{2} \mathrm{O}_{7}$ considering the doping study by Sato et al. [15]. It therefore seems plausible that the competition of FM/AFM interactions could be playing a crucial role in the formation of SG state in $\mathrm{Tb}_{2} \mathrm{Mo}_{2} \mathrm{O}_{7}$.

It is, however, possible that the presence of FM correlations is only a secondary effect and that the spin-glass/disorder relationship is more complex than some previous models allow. It is interesting to note that a similarly weak dependence of $T_{S G}$ on lattice disorder occurs in the La-substituted $\mathrm{Y}_{2} \mathrm{Mo}_{2} \mathrm{O}_{7}$ system discussed in the Introduction, where some evidence of FM correlations also exist. Recent theoretical work studying the role of disorder in geometrically frustrated antiferromagnetic systems has focused on how relatively little disorder can generate spin-glass freezing rather than an antiferromagnetically ordered phase $[11,28,29]$. In these studies, $T_{S G}$ is linear in the amount of disorder. A recent article by Shinaoka et al. [30] attempts to reconcile the apparent lack of strong 
dependence of $T_{S G}$ on lattice disorder by considering the role of spin-lattice coupling, finding that $T_{S G}$ can be pinned to a maximum value set by the presence of a nematic state [31]. Whether or not such a model is applicable, these studies clearly indicate that it is possible that spin-lattice or magnetoelastic coupling may play a very important role in the development of a spin-glass state.

\section{Conclusion}

This paper focuses on structural measurements of the $\mathrm{Tb}_{2} \mathrm{Mo}_{2} \mathrm{O}_{7}$ lattice. NPD data indicate both anisotropy and static (i.e. non-thermal) disorder on the Mo and $\mathrm{O}(1)$ sites. EXAFS results are in strong agreement, indicating static disorder in, primarily, the Mo-Mo and Tb-O(1) nearneighbor pairs. The measured order in the Tb-Mo pairs indicates the Mo-Mo disorder is mainly perpendicular to the the Tb-Mo pair directions, in excellent agreement with the ADPs measured with NPD. This disorder is similar to that measured previously in $\mathrm{Y}_{2} \mathrm{Mo}_{2} \mathrm{O}_{7}$, except that the amount of disorder is measurably less in $\mathrm{Tb}_{2} \mathrm{Mo}_{2} \mathrm{O}_{7}$. The better structural order is in contrast to the higher $T_{S G}$, indicating some other mechanism than nearest-neighbor AFM exchange must be playing an important role. More experimental probes should be applied to further elucidate the nature of this disorder. In particular, PDF experiments could help determine if the disorder in $\mathrm{Tb}_{2} \mathrm{Mo}_{2} \mathrm{O}_{7}$ manifests as any split pair distances. Such a detailed understanding of the local structural disorder should help determine the role of disorder in precipitating a spin-glass state in geometrically frustrated magnetic systems.

\section{Acknowledgment}

Ashfia Huq acknowledges the assistance of the POWGEN team: Jason Hodges, Olivier Gourdon and Luke Heroux. Research at Lawrence Berkeley National Laboratory was supported by the U.S. Department of Energy (DOE) under Contract No. DE-AC02-05CH11231. Research at Oak Ridge National Laboratory's Spallation Neutron Source was sponsored by the Scientific User Facilities Division, Office of Basic Energy Sciences, U.S. Department of Energy. The NIST Center for Neutron Research is in part funded by the National Science Foundation under Agreement No. DMR-0944772. X-ray absorption data were collected at Stanford Synchrotron Radiation Lightsource, a national user facility operated by Stanford University on behalf of the DOE, Office of Basic Energy Sciences.

\section{References}

[1] Gardner J S, Gingras M J P and Greedan J E 2010 Rev. Mod. Phys. 8253

[2] Mydosh J A 1993 Spin Glasses: An Experimental Introduction 1st ed (London: CRC Press)

[3] Ehlers G, Greedan J E, Stewart J R, Rule K C, Fouquet P, Cornelius A L, Adriano C, Pagliuso P G, Qiu Y and Gardner J S 2010 Phys. Rev. B 81224405

[4] Gardner J S, Gaulin B D, S-H Lee, Broholm C, Raju N P and Greedan J E 1999 Phys. Rev. Lett. 83211

[5] Keren A and Gardner J S 2001 Phys. Rev. Lett. 87177201

[6] Edwards S F and Anderson P W 1975 J. Phys. F: Metal Phys. 5965

[7] Sagi E, Ofer O, Keren A and Gardner J S 2005 Phys. Rev. Lett. 94237202

[8] Ofer O, Keren A, Gardner J S, Ren Y and MacFarlance W A 2010 Phys. Rev. B 82092403

[9] Booth C H, Gardner J S, Kwei G H, Heffner R H, Bridges F and Subramanian M A 2000 Phys. Rev. B 62 R755

[10] Greedan J E, Gout D, Lozano-Gorrin A D, Derahkshan S, Proffen T, Kim H J, Bozin E and Billinge S 2009 Phys. Rev. B 79014427 
[11] Saunders T E and Chalker J T 2007 Phys. Rev. Lett. 98157201

[12] Greedan J E, Reimers J N, Stager C V and Penny S L 1991 Phys. Rev. B 435682

[13] Apetrei A, Mirebeau I, Goncharenko I, Andreica D and Bonville P 2006 Phys. Rev. Lett. 97206401

[14] Singh D K, Helton J, Chu S, Han T H, Bonnoit C J, Chang S, Kang H J, Lynn J W and Lee Y S 2008 Phys. Rev. B $\mathbf{7 8} 220405$

[15] Sato M and Greedan J E 1987 J. Solid State Chem. 67 248-253

[16] Hanasaki N, Watanabe K, Ohtsuka T, Kézsmárki I, Iguchi S, Miyasaka S and Tokura Y 2007 Phys. Rev. Lett. 99086401

[17] Mirebeau I, Apetrei A, Goncharenko I, Andreica D, Bonville P, Sanchez J P, Amato A, Suard E, Crichton W A, Forget A and Colson D 2006 Phys. Rev. B $\mathbf{7 4} 174414$

[18] Hayes T M and Boyce J B 1982 Solid State Physics vol 37 (New York: Academic) p 173

[19] Li G G, Bridges F, Boyce J B, Claeson T, Ström C, Eriksson S G and Conradson S D 1995 Phys. Rev. B 51 8564-81

[20] http://lise.lbl.gov/RSXAP/

[21] Rodríguez-Carvajal J FULLPROF: A Program for Rietveld Refinement and Pattern Matching Analysis, at the Satellite Meeting on Powder Diffraction of the XV Congress of the IUCr, p.127, (1990) Toulouse, France

[22] Kennedy B J 1996 Mat. Sci. Forum 228-231 753

[23] Mirebeau I, Apetrei A, Rodríguez-Carvajal J, Bonville P, Forget A, Colson D, Glazkov V, Sanchez J P, Isnard $\mathrm{O}$ and Suard E 2005 Phys. Rev. Lett. 94246402

[24] Rule K C, Ehlers G, Stewart J R, Cornelius A L, Deen P P, Qiu Y, Wiebe C R, Janik J A, Zhou H D, Antonio D, Woytko B W, Ruff J P, Dabkowska H A, Gaulin B D and Gardner J S 2007 Phys. Rev. B 76212405

[25] Booth C H and Hu Y 2009 J. Phys.: Conf. Ser. 190012028

[26] Rehr J J, Albers R C and Zabinsky S I 1992 Phys. Rev. Lett. 693397

[27] Crozier E D, Rehr J J and Ingalls R 1988 X-Ray Absorption: Principles, Applications, Techniques of EXAFS, SEXAFS, XANES ed Konigsberger D and Prins R (New York: Wiley) p 373

[28] Bellier-Castella L, Gingras M J P, Holdsworth P C W and Moessner R 2001 Can. J. Phys. 791365

[29] Andreanov A, Chalker J T, Saunders T E and Sherrington D 2010 Phys. Rev. B 81014406

[30] Shinaoka H, Tomita Y and Motome Y (Preprint arXiv:1010.5625v1 [cond-mat.str-el])

[31] Shannon N, Penc K and Motome Y 2010 Phys. Rev. B 81184409 


\section{DISCLAIMER}

This document was prepared as an account of work sponsored by the United States Government. While this document is believed to contain correct information, neither the United States Government nor any agency thereof, nor the Regents of the University of California, nor any of their employees, makes any warranty, express or implied, or assumes any legal responsibility for the accuracy, completeness, or usefulness of any information, apparatus, product, or process disclosed, or represents that its use would not infringe privately owned rights. Reference herein to any specific commercial product, process, or service by its trade name, trademark, manufacturer, or otherwise, does not necessarily constitute or imply its endorsement, recommendation, or favoring by the United States Government or any agency thereof, or the Regents of the University of California. The views and opinions of authors expressed herein do not necessarily state or reflect those of the United States Government or any agency thereof or the Regents of the University of California. 